\title{
Die Entwicklung
}

\section{- \\ Lichtsinn und Farbensinn \\ in der Tierreihe}

(5)

\author{
Vortrag
}

gehalten bei der Versammlung deutscher Naturforscher und Ärzte in Wien am 25. September 1913

von

Carl Hess

in München

Mit 12 Abbildungen im Text.

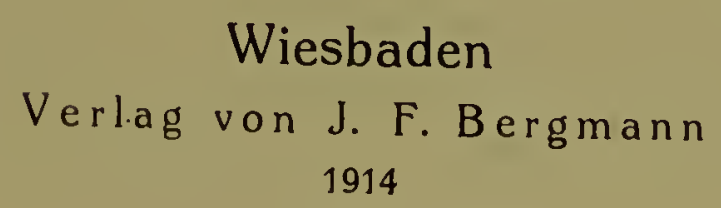


Nadhdruck verboten.

Übersctzungsrecht in alle Spradien vorbehalten. 
Der Lichtstrahl, der unser Auge trifft, weckt im optischen Empfangsapparate der Netzhaut, den Stäbchen und Zapfen, Regungen, die der Sehnerv dem Zentralorgan übermittelt; die hier ausgelösten Regungen treten als Helligkeit und als Farbe in unser Bewußtsein. Sind wir zu der Annahme berechtigt, daß diese Regungen und deren psychische Korrelate in allen Menschenaugen die gleichen sind? Daß ein Licht, das meinem Auge rot erscheint, auch den anderen Menschen so erscheine, gilt Vielen als selbstverständlich. Auch nachdem man erkannt hatte, daß nicht wenige Menschen die bunten Farben zum Teile wesentlich anders sehen, als der normale, glaubte man vielfach noch, zur Feststellung normalen Farbensinnes könne die zutreffende Bezeichnung der verschiedenen Farben durch den Untersuchten genügen. Es ist noch nicht lange her, daß wir wissen, wie unzulänglich der Aufschluß ist, den die Farbenbenennung durch einen Untersuchten uns über seine Sehqualitäten gibt, und daß Einem, der das Blut rot und das Blatt grün nennt, dennoch beide in wesentlich anderen Farben erscheinen können, als dem Normalen. Trotzdem dürfen wir, auch wenn ein Anderer uns durch Wort und Beschreibung nicht genügend Aufschluß über seine Sehqualitäten geben kann, doch annehmen, daß diese bei ihm keine wesentlich anderen sind als bei uns, sofern er unter verschiedenen, passend gewählten Versuchsbedingungen stets ähnliches oder das gleiche Verhalten zeigt wie der Normale.

Bedürfen wir aber der Farbenbenennung nicht, um Aufschluß über die Sehqualitäten eines anderen Menschen zu erhalten, so ist der Versuch gerechtfertigt, auch über die Sehqualitäten der Tiere Aufschluß zu bekommen, indem wir ihr Verhalten unter geeigneten Versuchsbedingungen jeweils mit dem eines unter 
entsprechende Bedingungen gebrachten Menschen vergleichen. In der Tat konnte ich so Ähnlichkeiten, Übereinstimmungen und Verschiedenheiten aufdecken, die uns in dem vielfach für dauernd verschlossen geltenden Gebiete zum Teile überraschende Aufschlüsse geben.

Bei einschlägigen Untersuchungen wurde man oft dadurch irre geführt, daß man zu den Versuchen fast ausnahmslos sich farbiger Gläser bediente, ohne Zusammensetzung und Lichtstärke der von diesen durchgelassenen Strahlungen genügend zu kennen. Erst geeignete Benützung der farbigen Lichter des Spektrums, deren relative Helligkeitswerte für das Menschenauge unter verschiedenen Bedingungen genügend bekannt sind, brachte mir bei systematischen Untersuchungen in der Tierreihe untereinander vergleichbare Ergebnisse. Es sind in erster Linie solche Untersuchungen am Spektrum, über welche ich im folgenden kurz berichte.

Über das Verhalten der Säugetiere im Spektrum mögen die folgenden Beispiele genügen: Wenn ich ein Spektrum auf einer horizontalen schwarzen Fläche entwerfe und auf ihr etwa weiße Reiskörner ausstreue, so erscheinen uns diese in den verschiedenen Farben des Spektrums und es ist nicht schwer, dessen Sichtbarkeitsgrenzen für unser Auge anzugeben. Ein vor die Fläche gebrachter Affe nimmt bald alle Körner vom roten bis zum violetten Ende, in genau der gleichen Ausdehnung, in der diese für uns sichtbar waren. Setze ich die Lichtstärke des Spektrums genügend herab und beobachte mit dunkeladaptirtem Auge, so sind jetzt für mich wesentlich nur die Körner in der Gegend des Gelbgrün bis Grün sichtbar; der gleich lange dunkeladaptirte Affe nimmt nur diese Körner, wiederum in der gleichen Ausdehnung, in der sie für uns sichtbar sind. Der Versuch lehrt, daß für den Affen das lichtstarke Spektrum am langwelligen und am kurzwelligen Ende merklich genau so weit reicht, wie für das Menschenauge, und $d a ß$ auch die hier in Betracht kommenden, durch Wechsel von Lichtstärke und Adaptation bedingten Änderungen dort offenbar im wesentlichen ähnliche oder die gleichen sind wie bei uns.

Setzen wir einen Tagvogel, etwa ein Huhn, vor ein solches lichtstarkes Spektrum, in dem Körner ausgestreut sind, so pidkt 
es diese am roten Ende genau so weit, als sie für unser Auge sichtbar sind, und nimmt außerdem die gelben und grünen Körner, dagegen läßt es die grünblauen, blauen und violetten unberührt, wiewohl sie für unser Auge gut sichtbar sind. Mit entsprechend abgeänderten Methoden konnte ich bei $\mathrm{S}$ childkröten nachweisen, daß auch für sie das Spektrum am kurzwelligen, violetten Ende hochgradig, bis zur Gegend des Blaugrün, verkürzt ist.

Die Erklärung für diese überraschende, in der ganzen Tierreihe nur bei Reptilien und $V$ ögeln auftretende Erscheinung gibt uns die mikroskopische Untersuchung der Netzhaut. Die Sauropsidenretina unterscheidet sich von jener der übrigen Wirbeltiere wesentlich dadurch, daß in ihren Zapfen zwischen Innen- und Außengliedern lebhaft gefärbte, meist gelbe oder rote sogenannte Ölkugeln gewissermaßen eingekeilt sind, so, daß zu den Außengliedern der Sehelemente vorwiegend nur die langwelligen Strahlen gelangen können, während die kurzwelligen in den farbigen Ölkugeln mehr oder weniger vollständig absorbiert werden. Wir lernen so die interessante Tatsache kennen, daß die fraglichen Sauropsiden die Welt der Farben etwa so sehen, wie wir, wenn wir unser Auge mit einem rötlichgelben Glase bewaffnen. Eine befriedigende Erklärung für das Auftreten dieser eigenartigen farbigen Gebilde läßt sich zurzeit noch nicht geben.

Unter den vielen Fragen, zu welchen diese neuen Befunde führen, sei hier nur die nach den S chmudkfärbungen der Vögel kurz berührt. Wenn die blauen Farben, die wir bei manchen Vogelarten, z. B. der Mandelkrähe, dem Eisvogel u. a. bewundern, wirklich, wie heute allgemein angenommen wird, Schmuckfarben sind, die zur Anziehung des anderen Geschlechtes dienen sollen, so müssen wir erwarten, daß bei diesen Vögeln die fraglichen farbigen Ölkugeln fehlen; denn einem Auge, in dem solche so reichlich und so lebhaft gefärbt sind, wie beim Huhn, wird auch ein für uns leuchtendes Blau höchstens bläulich grau oder aber farblos grau erscheinen. Findet man aber in den Netzhäuten solcher vorwiegend blauer Vögel die gelben und roten Ölkugeln in ähnlicher oder gleicher Weise wie beim Huhn, dann können hier die blauen Farben keine Schmudkfarben sein. Da auch 
sonst in der Tierreihe auftretende bunte Färbungen, wie wir sehen werden, vielfach zu Unredht als Schmudkfarben aufgefaßt werden, erscheint es von Interesse, daß uns hier ein Weg zur
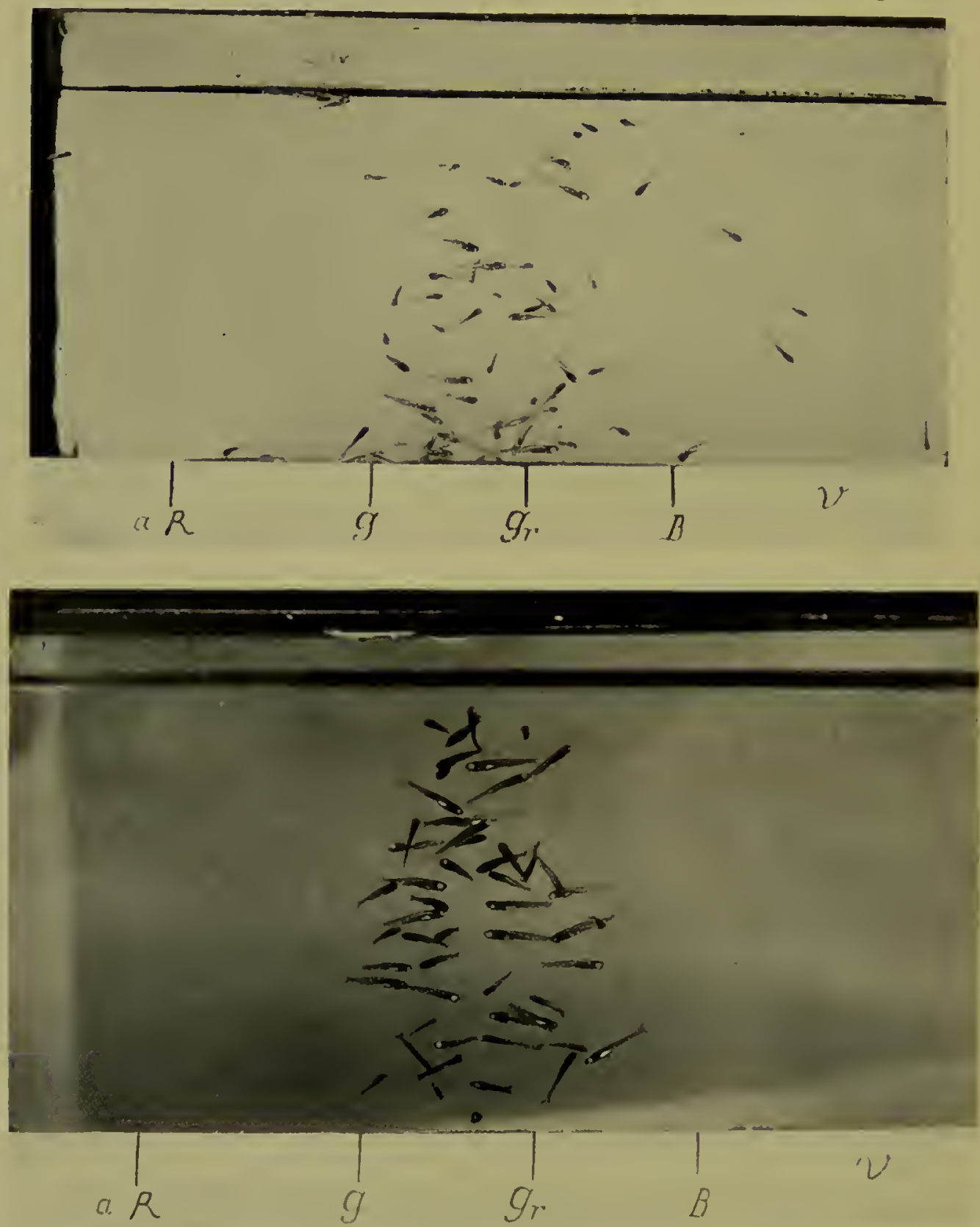

Fig. $1 \mathrm{a}$ und $1 \mathrm{~b}$.

Junge Weißfische in Spektrum; die Ansammlung in der Gegend des Gelbgrün bis Grün ist sehr deutlich. (Hier wie in den fólgenden Abbildungen bezeichnet a R. (= äußerstes Rot) das langwellige Spektrumende, G., Gr., B. die Gegend des reinen Gelb bzw. Grün u. Blau, V = Violett.) 
Beantwortung der Frage nach der Bedeutung der Färbungen gewisser Vogelarten gegeben ist.

Die bisher von mir untersuchten Amphibien zeigen farbigen Lichtern gegenüber ein Verhalten, das von jenem der Sauropsiden wesentlich verschieden ist, dagegen mit jenem des normalen Menschenauges weitgehende Übereinstimmung zeigt.

Ganz anders als die übrigen Wirbeltiere verhalten sich die von mir untersuchten Fis che. Füttert man solche Fischarten, die bei der Nahrungsaufnahme vorwiegend vom Auge geleitet werden, in geeigneter Weise im Spektrum, so nehmen sie am violetten Ende das Futter annähernd ebensoweit, als es für uns sichtbar ist, dagegen lassen sie das im Rot sichtbare Futter unberührt, selbst dann, wenn es dort unserem Auge sehr hell erscheint und entsprechend deutlich sichtbar ist. Das Spektrum ist also bei den Fischen, umgekehrt als wie bei den Sauropsiden, am roten Ende deutlich verkürzt. Da die Fischnetzhaut keine farbigen Ölkugeln enthält, ist der geringe Helligkeitswert roter Lichter für das Fischauge nicht, wie jener der blauen und violetten für das Vogelauge, durch Absorption zu erklären.

Zur Verfolgung der Frage nach dem Lichtsinne der Fische war die Entwicklung weiterer neuer Methoden erforderlich. Besonders geeignet erwies sich mir dabei die Eigentümlichkeit verschiedener Fische, stets zum Hellen zu schwimmen; diese Neigung ist insbesondere bei manchen Jungfischen sehr ausgesprochen, und bei vielen Arten können überraschend kleine Lichtstärkenunterschiede eines Reizlichtes genügen, um eine Ansammlung der Tiere in dem hellsten Teile ihres Behälters herbeizuführen. Setzt man solche Jungfische in einem geeigneten Gefäß den Strahlen eines Spektrums aus, so sammelt sich binnen wenigen Sekunden die große Mehrzahl der Fische in der Gegend des Gelbgrün bis Grün (Fig. 1); von hier nimmt ihre Zahl nach dem roten 'Ende rasch, nach dem violetten Ende langsamer ab. Schiebt man vor dem Behälter einen schwarzen Karton von der Seite her so vor, daß die Tiere z. B. vom Rot her allmählich in den Schatten kommen, so fliehen sie vor dem Schatten und sind in kurzer Zeit alle im Violett des Spektrums zusammengedrängt. Sowie der Karton weggezogen wird, machen die Fische rasch 
Kehrt und eilen wieder der Gegend des Gelbgrün bis Grün zu. Entsprechendes zeigt sich bei Vorschieben des Kartons vom Violett nach dem Rot hin. (Fig. 2.)

Wird die eine Hälfte des Behälters mit rotem, die andere mit blauem Lichte durchstrahlt, so schwimmen die Fische ins Blau (Fig. 3), auch wenn dieses unserem normalen, helladaptierten Auge dunkler erscheint als das Rot; wird jetzt ein schwarzer Karton vor die blaue Hälfte gebracht und diese somit verdunkelt, so

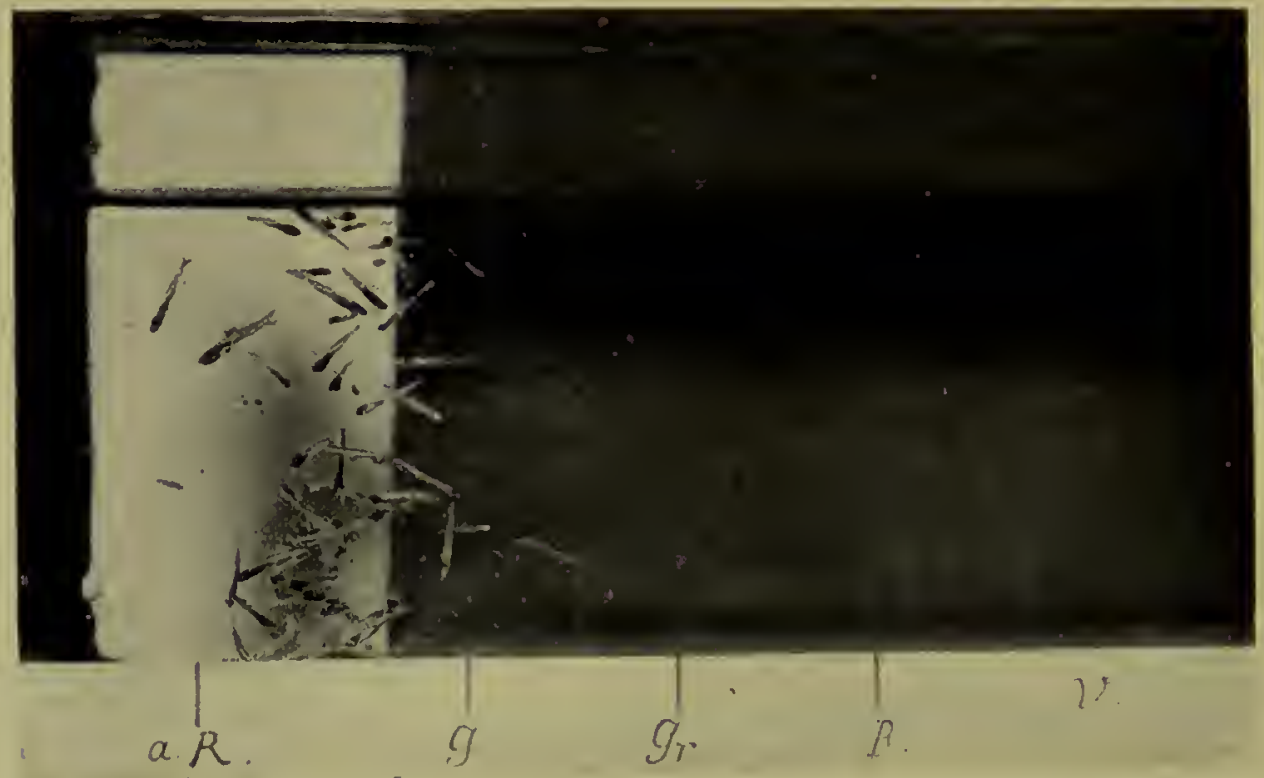

Fig. 2.

Junge Weißfische im Spektrum, durch Vorschieben eines schwarzen Kartons vom Violett her in der Gegend des Rot zusammengedrängt.

schwimmen die Fische ins Rot (Fig. 4). Das Gleiche ist der Fall, wenn bei unverdedktem Blau das Rot sehr lichtstark gemacht wird. Da$z$ wischen läßt sich leicht ein solches Lichtstärkenverhältnis zwischen Rot und Blau ermitteln, bei welchem die Fische sich angenähert gleichmäßig in beiden Behälterhälften verteilen.

Man hat angegeben, von den untersuchten Fischarten zeigten manche eine "Rotscheu"; sie sollten aus dem rot durchstrahlten in den dunklen Behälterteil fliehen. Es läßt sich aber leicht zeigen, daß diese Angaben auf Beobachtungsfehlern beruhen und daß die fraglichen Fische nie aus dem Rot ins Dunkel schwimmen. 
In Zusammenhang mit diesen irrigen Angaben über "Rotscheu" bei Fischen und zur Stütze derselben hatte auch die in Laienkreisen verbreitete Meinung, die Stiere zeigten eine "lebhafte Reaktion" auf Rot,

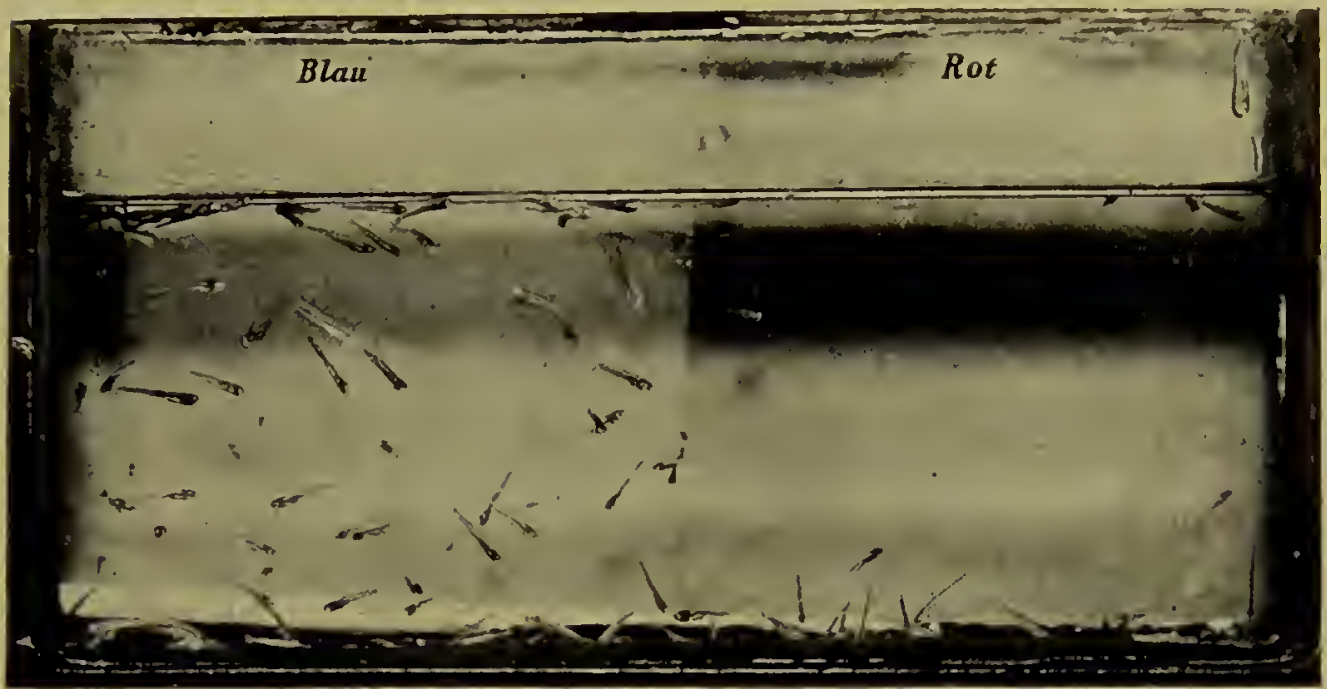

Fig. 3.

Fische in einem zur Hälfte mit Rot, zur Hälfte mit Blau durchstrahlten Behälter.

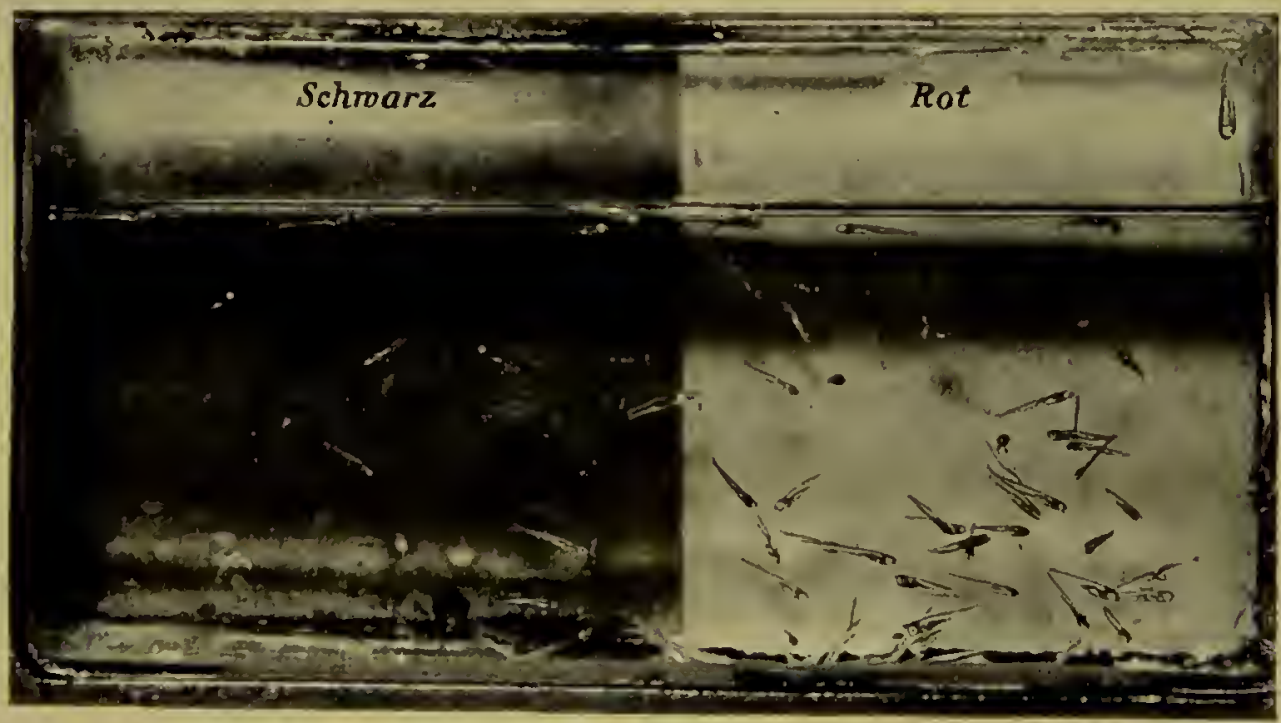

Fig. 4.

Die gleichen Fische, nachdem die vorher blau durchstrahlte Behälterhälfte mit einem schwarzen Karton verdedkt wurde.

in wissenschaftlichen Darstellungen Eingang gefunden. Dies veranlaßte mich, eine größere Zahl von Zuchtstieren in der Weise untersuchen zu laßen, daß große rote Tücher in verschiedener Weise dicht vor ihnen sichtbar gemacht wurden; wie für den mit den einschlägigen Verhältnissen genauer Vertrauten kaum anders $z u$ erwarten war, ergab sich, daß die 
rote Farbe den Stieren völlig gleidhgültig ist und keiner von ihnen dadurch irgendwie in Erregung versetzt wird.

Für ein normales Menschenauge ist das Spektrum in der Gegend des Gelb am hellsten; wenn also unsere Fische, die sonst stets zu der hellsten Stelle ihres Behälters schwimmen, im Spektrum das für uns deutlich weniger helle Gelbgrün bis Grün aufsuchen, so müssen wir entweder den Fischen eine "Vorliebe" für das Grün zuschreiben, oder es muß für ihr Auge das Spektrum an einer anderen Stelle am hellsten sein als für das normale Menschenauge. Die Annahme einer "Vorliebe" für Grün läßt sich leicht widerlegen; es genügt z. B., in unserem Spektrum in geeigneter Weise irgend eine Farbe genügend lichtstark zu machen, damit die Fischchen sofort in ihr sich sammeln. Auch mit anderen Methoden konnte ich die noch immer verteidigte Annahme einer "Vorliebe" der Fische für bestimmte Farben leicht widerlegen.

Prüfen wir die zweite Möglichkeit, daß für die Fische das Spektrum in der Gegend des Gelbgrün bis Grün am hellsten sei, so haben wir vor allem zu fragen, ob unsere Kenntnisse vom Lichtsinne des Menschen vielleicht Anhaltspunkte bieten, um ein soldhes Verhalten dem Verständnisse näher zu bringen. Wir wissen durch Ewald Hering, daß auch dem normalen Menschen im Spektrum die Gegend des Gelbgrün bis Grün dann am hellsten erscheint, wenn er ein genügend lichtschwaches Spektrum mit gut dunkeladaptiertem Auge betrachtet. Wir sehen dieses dann als farblos graues Band, das im Gelbgrün bis Grün am hellsten ist, und dessen Helligkeit von hier nach dem kurzwelligen Ende langsam abnimmt, sehr rasch dagegen nach dem dem Rot entsprechenden Ende; die Gegend des Rot erscheint dem gut dunkeladaptierten Menschenauge in einem solchen lichtschwachen Spektrum tief dunkelgrau, fast schwarz, das Spektrum ist also für ihn jetzt am langwelligen Ende verkürzt.

Weiter zeigte Hering, daß der total farbenblinde Mensch, dem die ganze Welt der Farben snur in verschiedenen Abstufungen farbloser Helligkeiten erscheint, das Spektrum bei jeder Lichtstärke und bei jedem Adaptationszustande so sieht, wie der dunkeladaptierte Normale das lichtschwache Spektrum (Fig. 5). 
Durch Aufsuchen passender Gleidhungen zwischen verschiedenen farbigen und zwischen farbigen und angenähert farblosen Lichtern von meßbar variabler Lichtstärke vermochte ich nun auf mehreren Wegen zu zeigen, daß die farbigen Lichter des Spektrums für die Fische die gleichen relativen Helligkeitswerte haben, wie für den total farbenblinden Menschen. Mit anderen Worten, es verhielten sich bei allen meinen Versuchen die Fische so, wie es der Fall sein muß, wenn ihre Sehqualitäten jenen des total farbenblinden

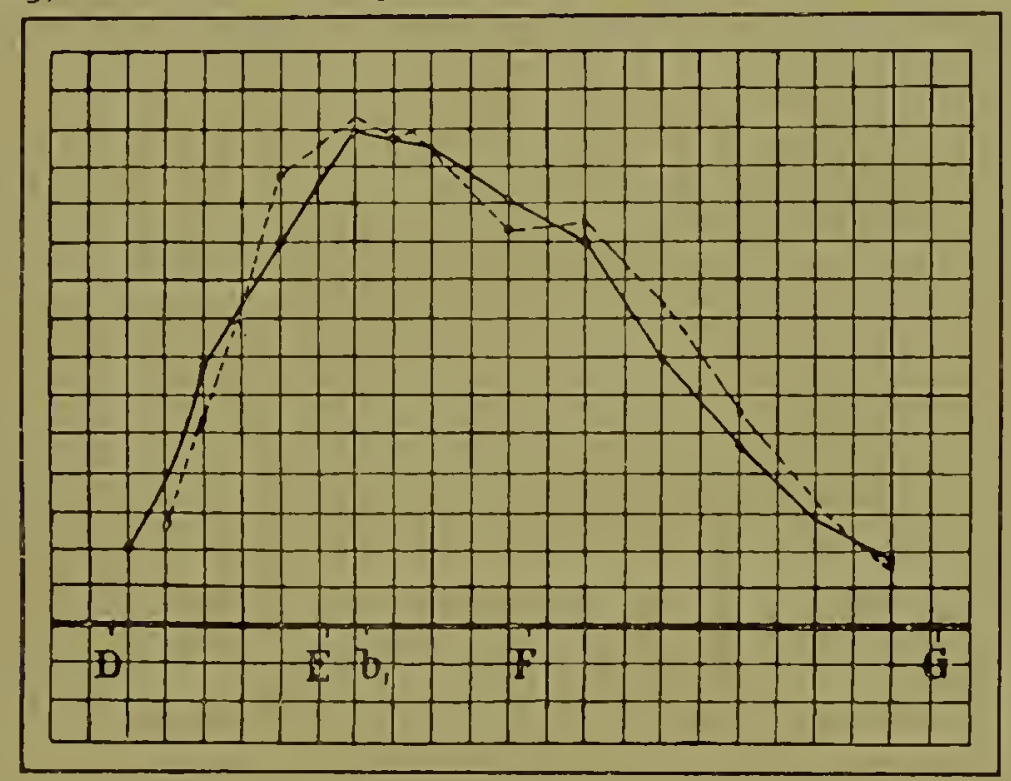

Fig. 5.

Kurven der relativen Helligkeiten in Spektrum für einen total farbenblinden (ausgezogene Linie) und für einen dunkel adaptierten normalen Menschen (punktierte Linie). (Nach E. Hering.)

Menschen ähnlich oder gleich sind. Unter der Voraussetzung, daß die Fische die Welt der Farben im wesentlichen so sehen, wie ein total farbenblinder Mensch, läßt sich für jede beliebige Kombination zweier farbiger Reizlichter im voraus angeben, wie sich die zum Hellen schwimmenden Tiere den beiden Lichtern gegenüber verhalten werden.

Die Zoologen schreiben den Fischen noch ziemlich allgemein einen Farbensinn zu. Ich erinnere hier nur daran, daß weite Kreise die bunten Farben vieler Fische als "auf das Auge berechneten" Schmuck deuten und daß auch in der Angelfischerei die Farbe des benützten Köders vielfach für sehr wichtig gilt. So wedkte 
das eben mitgeteilte Ergebnis meiner Untersuchungen lebhaften Widerspruch und gab Anlaß zu dem Versuche, die übliche Annahme eines Farbensinnes bei Fischen durch neue Beobachtungen zu stützen. Es wurde z. B. angegeben, die in unseren Gewässern häufige Elritze müsse Farbensinn haben, weil sie auf gelbem und rotem Grunde stets gelbe Färbung annehme, die auf farblosem Grunde wieder verschwinde. Tatsächlich erfolgt aber eine solche Farbenanpassung gar nicht, die einschlägigen Angaben beruhen auf Beobachtungsfehlern. Die Farbe des Grundes ist ohne jeden Einfluß auf die individuell mannigfach wechselnde Färbung der Elritze.

Größeres Interesse beansprucht die Lehre vom sogenannten Hochzeitskleid der Fische. Es ist lange bekannt, daß insbesondere bei den Männchen verschiedener Fische Bauch und Bauchflossen zur Laichzeit sich gelbrot oder tief rot färben. Man sieht hierin vielfach einen zur Anziehung der Weibchen bestimmten Schmudk, wiewohl noch nicht bekannt ist, ob bei den Fischen überhaupt eine Wahl von seiten der Weibchen stattfindet. Die fragliche Annahme gründet sich weiter auf die irrige Voraussetzung, die Farben, die wir an den Fischen in Luft wahrnehmen, müßten in gleicher Weise bei den im Wasser verschieden weit unter der Oberfläche schwimmenden Tieren sichtbar sein. Es wurde nie untersucht, inwieweit die grüne bzw. blaue Färbung des Wassers durch Absorption der langwelligen Strahlen die Wahrnehmung des in Luft an den Fischen sichtbaren Gelb und Rot in ihrer gewohnten Umgebung erschwert bzw. unmöglich macht.

Die in Frage stehenden sog. Hochzeitsfarben finden sich hauptsächlich an der Bauchseite der Fische, die vorwiegend nur von den verhältnismäßig geringen Lichtmengen getroffen wird, welche aus den tieferen Wasserschichten zurüdkkommen und wesentlich nur von den dem Grün bis Blau des Spektrums entsprechenden Strahlen gebildet werden. Sowohl hinsichtlich der Farbe selbst als hinsichtlich ihres Auftretens am Fischbauche wären also Gelb und Rot für die Wahrnehmung besonders ungeeignete Färbungen und schon um deswillen war die Deutung, daß es sich um Schmudkfarben handle, wenig wahrscheinlich. Die endgültige Widerlegung der üblichen Annahme ergibt sich aber aus folgendem: 
Ich konnte unschwer nachweisen, daß (z. B. in vershiedenen von mir untersuchten Seen) schon 6-8 m unter der Wasseroberfläche das Gelbrot des Fischbauches von einem dort befindlichen farbentüchtigen Menschenauge nicht mehr als Farbe wahrgenommen werden kann. Von den Fischen, bei welchen das fragliche Hochzeitskleid vielfach beobachtet wird, laichen manche Arten in Tiefen von $20 \mathrm{~m}$ und mehr, wo also von jenen roten und gelben Farben sicher nichts mehr sichtbar ist; der Königseesaibling, der eine sehr lebhafte Rotfärbung zeigt, laicht sogar in Tiefen von $60 \mathrm{~m}$. Damit ist ganz unabhängig von der Frage nach einem Farbensinne bei Fischen der streng physikalische Nachweis von der Unhaltbarkeit der herrschenden Lehre erbracht, wonach die bunten Farben, die diese Fische zur Laichzeit zeigen, ein auf das Auge der Weibchen berechneter Schmuck sein sollten.

Es ist bisher nicht eine einzige Tatsache bekannt geworden, die auf einen Farbensinn bei Fischen deutete. Daß die bisher untersuchten Fische sich durchaus so verhielten, wie unter entsprechende Bedingungen gebrachte total farbenblinde Menschen, kann nur auf den ersten Blick befremdlich erscheinen: Wer Entwidklung und Funktion der Sinnesorgane der Lebewesen in Zusammenhang mit den besonderen Existenzbedingungen der letzteren $\mathrm{zu}$ verstehen bemüht ist, erkennt bald, wie geringe Vorteile ein dem unsrigen ähnlicher Farbensinn den Fischen, wie den Wassertieren überhaupt, bringen könnte. Erscheint doch auch dem farbentühtigen Menschenauge schon wenige Meter unter der Wasseroberfläche die ganze Umgebung vorwiegend blau oder grün und in ihren Einzelheiten viel mehr nach Helligkeiten als nach Farben verschieden.

Der geringe Helligkeitswert, den die langwelligen, für uns vorwiegend roten Lichter für das Fischauge haben, entspricht der geringen biologischen Bedeutung, die diese Strahlungen im allgemeinen infolge ihrer starken Absorption im Wasser für die Fische haben können. Die kurzwelligen Lichter dringen in viel größere Tiefen, doch nimmt auch bei ihnen die Lichtstärke mit zunehmender Tiefe beträchtlich ab. Es muß daher für die Fische, die bald näher zur Oberfläche, bald mehr in der 
Tiefe schwimmen, von großer Bedeutung sein, daß ihre Sehorgane sich diesen wechselnden Lichtstärken anzupassen vermögen; und in der Tat konnte ich bei verschiedenen Fischarten ausgiebige adaptative Änderungen nadiweisen, die nach Art und Umfang weitgehende Ähnlichkeit mit jenen im Menschenauge zeigen.

$\mathrm{Zu}$ den erwähnten Lichtsinnuntersuchungen erweisen sich nicht alle Fischarten gleich gut geeignet. Bestimmte Beobachtungsreihen lassen sich nur bei solchen Arten vornehmen, die ihrer Nahrung vorwiegend oder ausschließlich mit Hilfe der Augen nachgehen. Man nahm bisher viel-

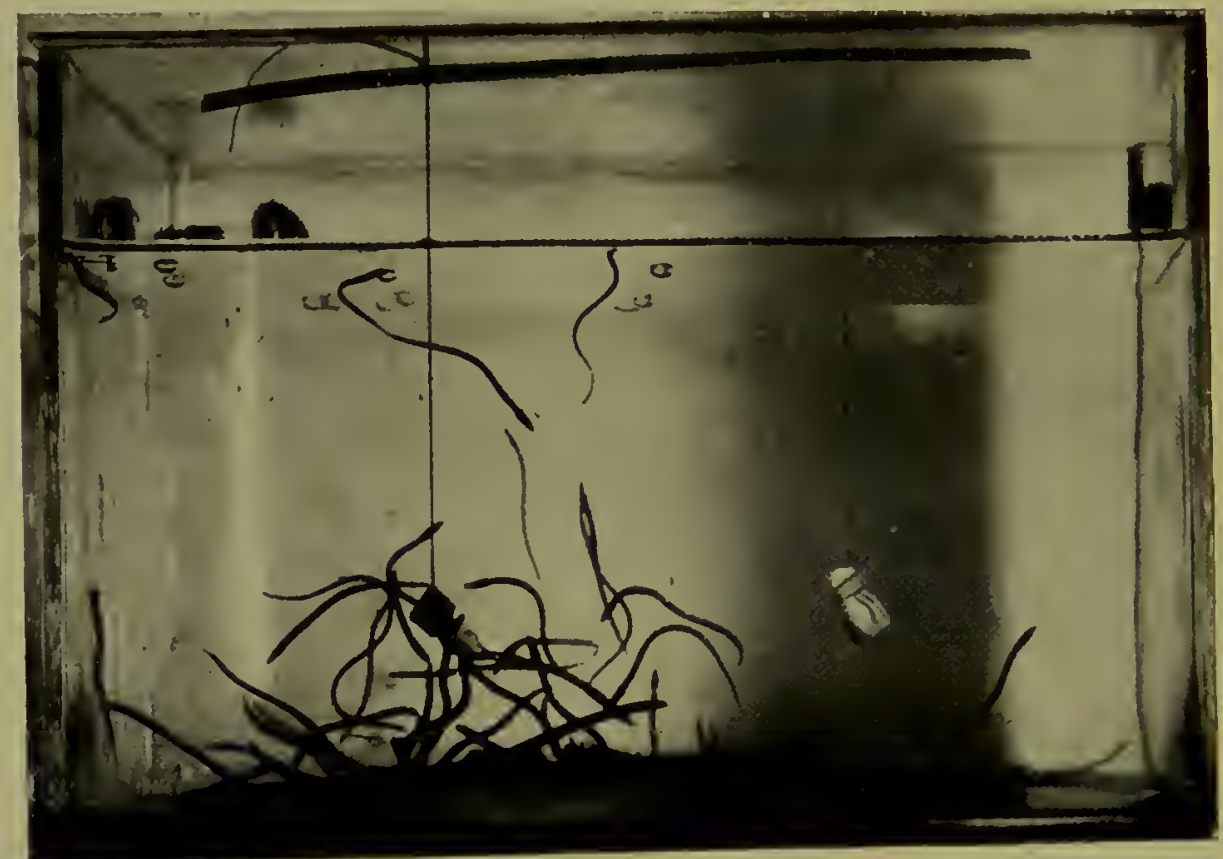

Fig. 6.

Junge Aale, die sich um ein mit Fleisch gefülltes Säckchen zusammendrängen, ein gleiches, leeres aber unbeachtet lassen.

fach an, daß solches bei Knochenfischen allgemein der Fall sei, was indessen nicht zutrifft. Es ist eine Reihe von Teleosteern bekannt, bei welchen der Geruch für das Auffinden der Nahrung von größter Bedeutung ist; für Aale konnte ich solches duich folgenden Versuch nachweisen: In ein Aquarium mit jungen Aalen werden zwei gleiche Leinwandsädkchen gebradit, das eine leer, das andere mit Fleisch gefüllt; in kürzester Zeit schwimmen fast alle Aale in sichtlicher Erregung um dieses letztere und lassen das leere Sädkchen unbeachtet.

So, wie in diesem letzteren Falle wird wohl auch sonst die Richtung der Schwimmbewegungen insbesondere der Jungfische zu einem großen Teile durch den Nahrungstrieb bestimmt, und 
die Vermutung liegt nahe, daß auch jene Ansammlung der Fische an den hellsten Stellen ihrer Umgebung mit hierauf zurückzuführen ist: denn die winzigen Gebilde und Lebewesen, die den Fischen zur Nahrung dienen, müssen im allgemeinen für sie um so besser sichtbar sein, in je hellerer Umgebung sie erscheinen, (womit nicht gesagt sein soll, daß nicht auch noch andere Umstände auf jene Ansammlung im Hellen von Einfluß sein können).

Man wollte die Bewegungen der Fische und verschiedener anderer Tiere zum Lichte als "Laboratoriumsprodukt" und als "Fluchterscheinung" deuten. Aber wir brauchen uns nur der bekannten Erfahrung der Fischer zu erinnern, zu deren Lichtquellen des Nachts Fische und Krebse von weit her auch aus ihrer gewohnten Umgebung herankommen, um uns aufs neue zu über zeugen, daß jener mächtige Drang zum Lichte, der bei Wasserund bei Lufttieren in so mannigfach verschiedenen Formen wiederkehrt, weder als Flucht, noch als eine künstlich erzeugte Reaktion aufgefaßt werden darf, vielmehr einen ursprünglichen, tief eingewurzelten Trieb darstellt, dem wir für das Leben der betreffenden Arten auch da eine große Bedeutung zuerkennen müssen, wo unsere biologischen Kenntnisse zur Aufdeckung einer solchen heute noch nicht ausreichen. -

Bei dem niedersten Wirbeltiere, dem Amphioxus, dessen Sehorgane lediglich von einzelnen, über den ganzen Körper verstreuten Zellen gebildet werden, ruft Belichtung fluchtartige Bewegungen hervor, deren Lebhaftigkeit mit der Lidhtstärke des Reizlichtes wächst. Für die verschiedenen Lichter des Spektrums fand id, daß die gelbgrünen bis grünen die lebhaftesten Bewegungen auslösen, die blauen etwas weniger lebhafte, während die gelbroten und roten nahezu oder ganz wirkungslos sind. Also auch hier schon kommen den verschiedenen farbigen Lichtern die gleichen relativen Reizwerte $z u$, wie bei den übrigen Fischen und beim total farbenblinden Menschen.

Die bei den Fischen gefundenen Lichtreaktionen geben uns den Schlüssel zum Verständnisse der unter dem Einfluß des 
Lichtes eintretenden Lebensäußerungen bei zahlreichen im Wasser lebenden Wirbellosen. So mannigfach verschieden die Art dieser Reaktionen bei den verschiedenen Tierarten ist, überall begegnete ich der gleichen Abhängigkeit von der Wellenlänge wie bei den Fischen: auch alle bisher untersuchten Wirbellosen verhalten sich in allen von mir ermittelten Beziehungen so, wie unter entsprechende Bedingungen gebrachte total farbenblinde Menschen sich verhalten würden.

Einige wenige Beispiele müssen genügen.

Für zahlreiche Krebsarten fand ich lebhafte Neigung, zum Hellen zu schwimmen: Setzt man einige hundert Podopsis (kleine,

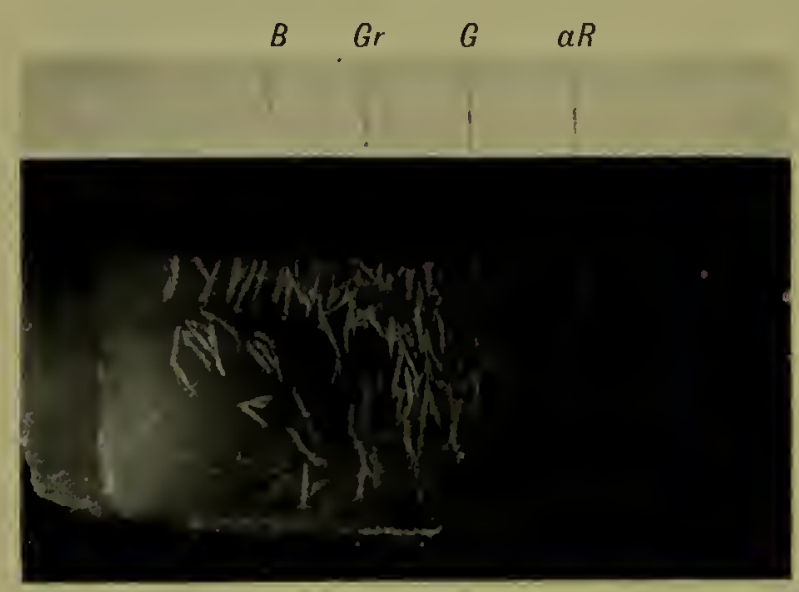

Fig. 7.

Podopsis im Spektrum. lebhaft schwimmende marine Krebse) den Strahlen eines Spektrums aus, so sammeln auch sie sich in wenigen Sekunden vorwiegend in der Gegend des Gelbgrün bis Grün, ganz so, wie wir es bei den Fischen sahen. Ein Gleiches läßt sich für junge Cephalopodenlarven nachweisen. Hier ist von besonderem Interesse, daß bereits Embryonen, die noch 2-3 Wochen vor der Reife entfernt sind, sobald man sie aus ihrer Hülle befreit, rasch mit lebhaften Bewegungen der Gegend des Gelbgrün bis Grün des Spektrums zueilen. So lange vor der physiologischen Geburt vermag hier das Sehorgan bereits die Helligkeitsempfindungen zu vermitteln, die zu jenen komplizierten, wohl koordinierten Schwimmbewegungen führen.

Hier, bei den Cephalopoden, konnte ich die Frage nach der Art ihrer Helligkeitsempfindung noch auf einem zweiten, von dem ersten ganz unabhängigen Wege in Angriff nehmen. Von allen Wirbellosen haben die Kopffüßer allein ein wesentlich durch die Belichtung bestimmtes, der Regulierung der einfallenden 
Lidhtmengen dienendes Pupillenspiel, dessen Lebhaftigkeit die Tiere für unsere $Z$ wecke besonders geeignet macht. Photographische Aufnahmen einer Sepia, die ich in passender Weise an verschiedene Stellen eines Spektrums brachte, zeigen, daß die Pupille unter der Wirkung der verschiedenen farbigen Lichter sich in verschiedenem Maße verengt, und man erhält so unschwer einen Überblick über die pupillomotorischen Reizwerte der verschiedenen farbigen Strahlungen. Auch hier haben die gelbgrünen und grünen Lichter den größten motorischen Reizwert, die Pupille wird durch sie am meisten verengt, die gelbroten

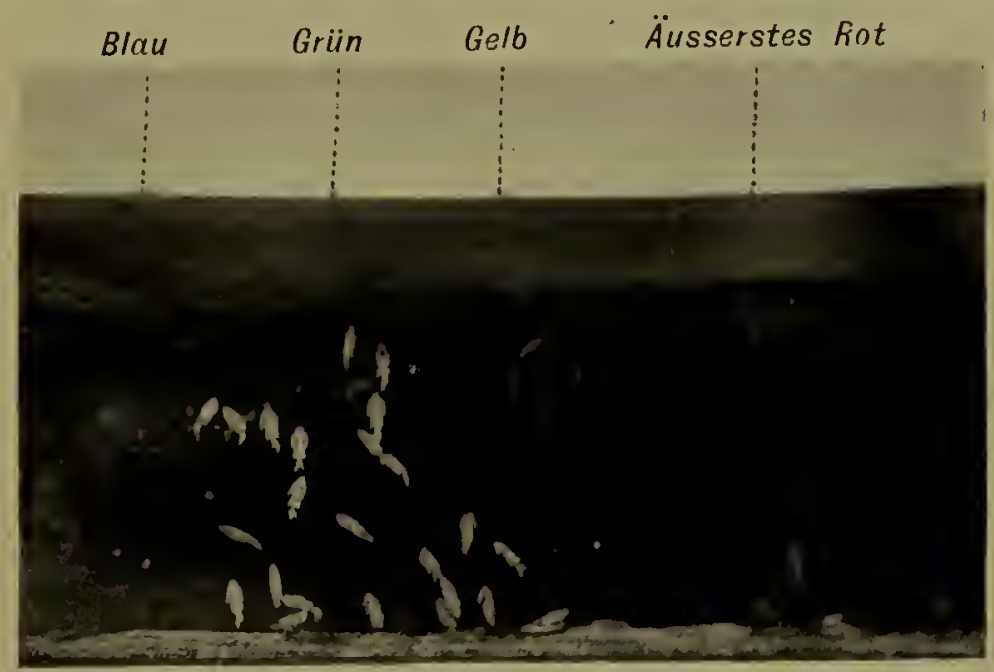

Fig. 8.

Embryonen von Cephalopoden (Loligo) im Spektrum.

und roten Lichter sind fast unwirksam, die Pupille bleibt bei Belichtung mit ihnen fast eben so weit, wie sie im Dunkeln war, während das für uns viel weniger helle Blau des gleichen Spektrums eine stärkere Verengerung auslöst, als jenes für uns verhältnismäßig helle Rot (Fig. 9).

Im normalen Menschenauge finden wir das Maximum der Pupillenverengerung im Gelb des Spektrums, dagegen zeigt die Pupille des total farbenblinden Menschenauges hinsichtlich der relativen motorischen Reizwerte der verschiedenen farbigen Strahlungen weitgehende Übereinstimmung mit dem Verhalten der

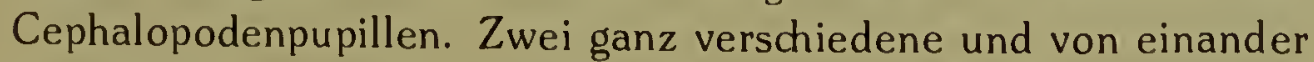
unabhängige Wege führen also hier zu ganz übereinstimmenden Ergebnissen hinsichtlich des Lichtsinnes der Kopffüßer. 
Man hat früher und auch in jüngster Zeit wieder versucht, der Frage nach dem Sehen der Kopffüßer durch das Studium der bei Belichtung der Netzhäute ausgeschnittener Augen wahrnehmbaren „Aktionsströme“ näher zu kommen. So interessant solche Versuche für die Kenntnis der Änderungen sind, die unter dem Einflusse des Lichtes in der Netzhaut eintreten können, von den durch jene Änderungen im Gehirn bedingten Vorgängen und insbesondere von den an letztere gebundenen Lich te mp find ungen erfahren wir auf diesem Wege nichts. Über den Licht- und Farbensinn der Tiere,

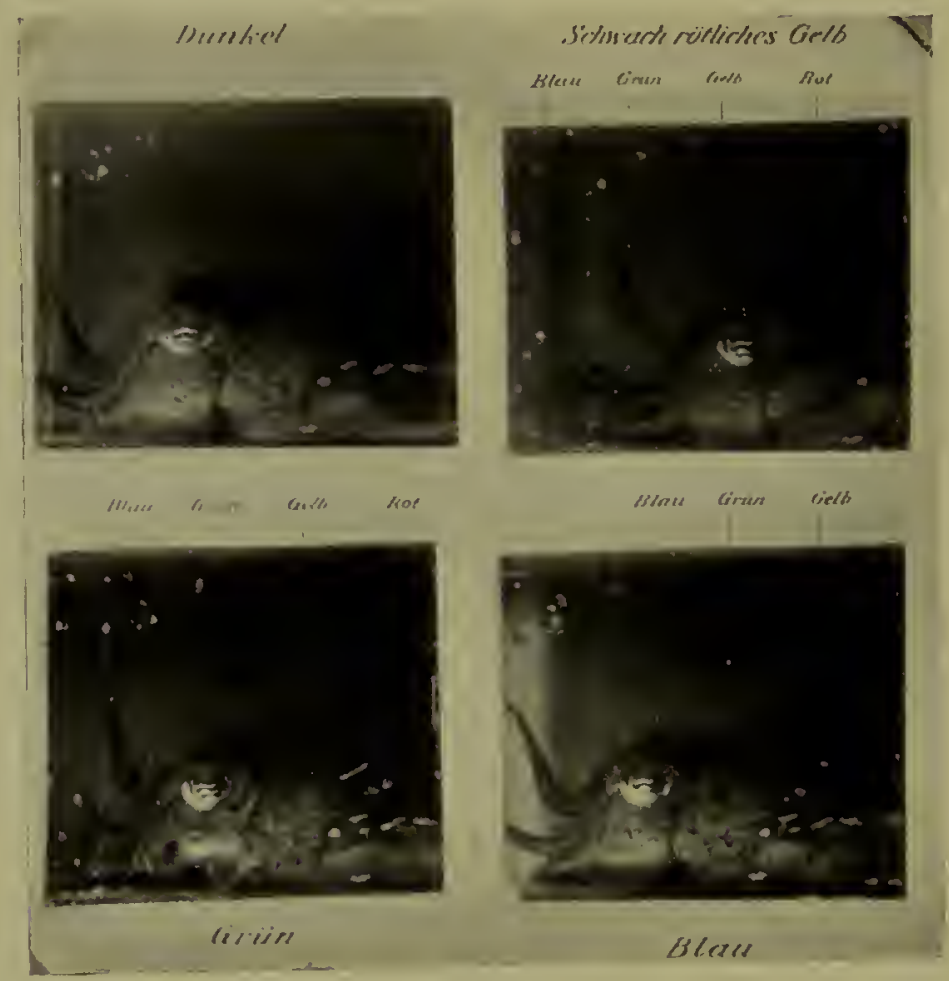

Fig. 9.

Eine Sepia in verschiedenen Lichtern des Spektrums.

der uns hier in erster Linie beschäftigt, haben die Aktionsströme bisher keine verwertbaren Aufschlüsse bringen können.

Wie die von mir mit spektralen Lichtern angestellten Messungen über wichtige Fragen aus der vergleichenden Physiologie des Gesichtssinnes Aufschluß geben können, sei noch an dem folgenden Beispiele dargetan. Die lichtempfindlichen Elemente der Netzhaut, die Stäbchen und Zapfen, werden nach außen von einer mit feinsten gelbbraunen Farbkörnchen erfüllten Zelllage begrenzt; bei Belichtung wandern diese Körnchen bei Fischen und 
Cephalopoden in der Richtung nach dem Glaskörper eine Strecke weit vor, so, daß in länger belichteten Fischaugen die Außenglieder jedes einzelnen Sehelementes von einem mehr oder weniger dichten Mantel aus solchen braunen Farbkörnchen umhüllt sind. Dies hat zur Folge, daß das "terminale", d. i. das zu den Außengliedern selbst gelangende Licht in lange dunkel gehaltenen Fischaugen von anderer physikalischer Zusammensetzung ist als in lange belichteten; denn in letzteren halten die vorgewanderten Farbkörnchen mehr oder weniger große Mengen insbesondere von den kurzwelligen, blauen und violetten Strahlen zurück. Auch dieser Einfluß der Pigmentwanderung auf die Zusammensetzung des terminalen Lichtes läßt sich mit den von mir entwickelten Methoden leicht nachweisen und bis zu einem gewissen Grade messend verfolgen.

Betrachten wir bei hellem Tageslichte eine rote und eine blaue Fläche und setzen nun die Lichtstärke allmählich herab, wobei wir unser Auge an das umgebende Dunkel anpassen, so zeigt sich bald eine auffällige Änderung der Helligkeit, in welcher uns die beiden farbigen Flächen erscheinen; das Rot, das wir mit dem an Tageslicht adaptierten Auge sehr hell und beträchtlich heller als das Blau gesehen hatten, erscheint bei entsprechend herabgesetzter Lichtstärke dem dunkeladaptierten Auge tief dunkel grau, fast schwarz und viel dunkler als das nunmehr gleichfalls farblos, aber ziemlich hell grau gesehene Blau. Diese bei Änderung von Lichtstärke und Adaptation eintretenden Änderungen der relativen Helligkeiten farbiger Lichter sind unter dem Namen des Purkinje'schen Phänomens bekannt. Für das total farbenblinde Auge treten die fraglichen Änderungen nicht ein, bei ihm fehlt also das Purkinje'sche Phänomen. Es muß daher auch auf diesem Wege möglich sein, die Richtigkeit meiner früher mit anderen Methoden bei Fischen und Wirbellosen erhobenen Befunde zu prüfen.

In der Tat konnte ich nachweisen, daß bei den Fischen die dem Purkinje'schen Phänomen entsprechenden Helligkeitsänderungen bei Änderung von Lichtstärke und Adaptation nicht auftreten.

Man hat irrtümlicherweise gewisse Verschiedenheiten des Verhaltens lange hell und lange dunkel gehaltener Fische als Purkinje- 
sches Phänomen deuten wollen: man übersah dabei die vorher besprochenen, durch die Pigmentwanderung bedingten Unterschiede der physikalischen Verhältnisse bei Hell- und bei Dunkelfischen, die im Menschenauge nicht in Betracht kommen, da hier eine phototrope Pigmentwanderung fehlt.

Dem gleichen prinzipiellen lrrtume begegnen wir in Untersuchungen aus der jüngsten Zeit über den Lichtsinn der Kopffüßer; auch bei diesen wandern die hier gleichfalls reichlich vorhandenen Farbstoffkörnchen bei Belichtung glaskörperwärts vor, vielfach in so beträchtlichen Mengen, daß ich die Netzhäute lange belichteter Augen oft in großer Ausdehnung tief braun,

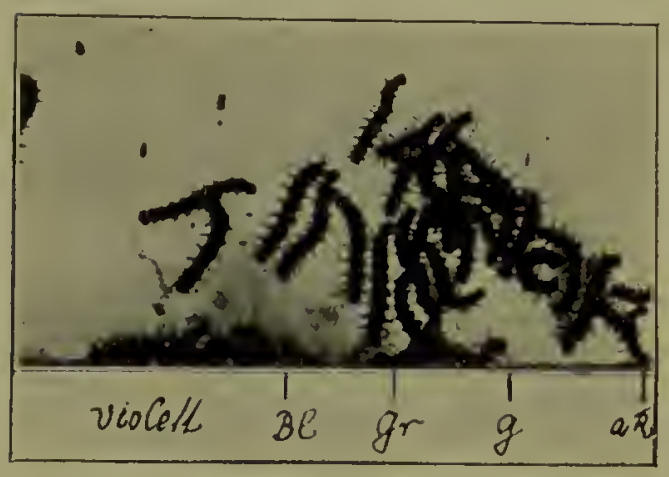

Fig. 10.

Raupen (Porthesia) im Spektrum. die lange dunkel gehaltener dagegen hellgrau fand.

Es war danach schon auf Grund meiner früheren $\mathrm{Be}$ funde an Fischen mit Bestimmtheit vorauszusagen, daß auch bei den Kopfüßern das terminale Licht in lange hell gehaltenen Augen eine verhältnismäßig viel schwächere Wirkung der blauen und violetten Strahlen zeigen würde, als in lange dunkel gehaltenen. In der Tat konnte man solches bei neueren Versuchen mit Aktionsströmen nachweisen, hat aber auch hier die einschlägigen Erscheinungen irrtümlich als Purkinje'sches Phänomen gedeutet, von dem sie nach dem Gesagten grundverschieden und scharf zu trennen sind. Tatsächlich verhalten sich die Kopffüßer in allen bisher festgestellten Beziehungen so, wie unter entsprechende Bedingungen gebrachte total farbenblinde und durchaus anders, als wie farbentüchtige Menschen sich verhalten würden. -

Wir sprachen bisher nur von den durch Belichtung veranlaßten Bewegungen bei in Wasser lebenden Wirbellosen; ein ähnliches oder gleiches Verhalten konnte ich für eine Reihe von Raupen, Mücken, Fliegen und Käfern nachweisen, die gleichfalls zum Teil ausgesprochene Neigung zeigen, zum Hellen zu gehen und auf 
genügend kleine Lichtstärkenunterschiede mit entsprechenden Kriech- oder Fliegbewegungen reagieren. Die Raupen von Porthesia z. B. bleiben, so lange sie sich im Dunkeln befinden, meist träge am Boden ihres Behälters liegen, beginnen aber bei Belichtung mit Tageslicht bald nach oben zu kriechen, um so früher und um so lebhafter, je größer die Lichtstärke ist; bringen wir die Tiere in passender Weise in ein Spektrum, so kriechen zunächst vorwiegend jene im Gelbgrün und Grün nach oben, weniger lebhaft die im Blau, am wenigsten die im Rot des Spektrums befindlichen (vgl. Fig. 10).

Über die interessanten Lichtreaktionen bei unserer Stechmücke müssen einige kurze Andeutungen genügen: Auch für sie fand ich, daß sie stets lebhaft nach der für sie hellsten Stelle ihres Behälters, im Spektrum nach der Gegend des Gelbgrün bis Grün eilt. Diese Mücken tanzen bekanntlich oft des Abends in Schwärmen einige Meter über der Erde und der Schwarm folgt gelegentlich einem langsam gehenden Menschen eine Strecke weit; auch diese Bewegungen der Mückenschwärme fand ich wesentlich durch die Helligkeit der unter ihnen befindlichen Gegenstände bestimmt: Bewegte ich z. B. geeignete große hellund dunkelgraue und verschiedenfarbige Flächen wagerecht langsam unter solchen Schwärmen hin und her, so folgten letztere stets den weißen und hellgrauen, nicht aber den dunkelgrauen und schwarzen Flächen; blauen und grünen Flächen folgten sie ebenso wie den hellgrauen, roten dagegen ebensowenig wie den dunkelgrauen und schwarzen, auch wenn unserem normalen Auge das Rot viel heller erschien als das Blau. Kurz, auch hier ist das Verhalten der Tiere ganz so, wie das eines unter entsprechende Bedingungen gebrachten total farbenblinden, und durchaus anders, wie das eines farbentüchtigen Menschen.

Andere Reaktionen zeigen die im Wasser lebenden Larven dieser Mücken; sie hängen sich, wenn sie nicht gestört werden, in großer Zahl mit ihren Atemröhren an der Wasseroberfläche an, fliehen aber schon bei sehr unbedeutenden Lichtstärkenabnahmen rasch nach unten. lhre Empfindlichkeit für Lichtstärkenunterschiede ist so groß, daß sich überraschend genaue Messungen vornehmen und ohne besondere Hilfsmittel, allein mit passenden 
farbigen Papieren, leicht Versuche anstellen lassen, die auch dem Laien eine gute Vorstellung von den merkwürdigen, für unsere Kenntnis von den Helligkeitsempfindungen jener Insekten so wichtigen Reaktionen geben können.

Wie bei den Fischen werden wir auch bei den Insekten dem hier gleichfalls so weit verbreiteten Drange zum Hellen eine wesentliche biologische Bedeutung zuerkennen müssen. Man hat sich viel bemüht, eine Erklärung insbesondere für die so außerordentlich unzweckmäßig erscheinende Tatsache zu finden, daß zahlreiche Nadhtinsekten in die Flamme fliegen und so den Tod finden. Eine eingehendere Besprechung der zum Teile recht wenig befriedigenden Erklärungsversuche - auch hier wollte man das Fliegen in die Flamme als "Flucht"erscheinung auffassen - kann nidht meine Aufgabe sein. Die folgenden Andeutungen mögen genügen, um die Richtung $z u$ zeigen, in der nach meiner Meinung die Erklärung jener Erscheinungen zu suchen ist.

Die fraglichen Insekten besitzen sämtlich gut entwickelte, zum Teile verhältnismäßig große Sehorgane. Für Nachtvögel konnte ich durch messende Untersuchungen dartun, daß die Sichtbarkeitsgrenze vor ihren Augen bewegten Futters bei allmählich abnehmender Lichtstärke keine wesentlich andere ist, als für unser gut dunkeladaptiertes Auge. Auch die nächtlichen Insekten vermögen jedenfalls in völliger Dunkelheit nichts $z u$ sehen; damit ihre Sehorgane überhaupt verwertbare Eindrücke vermitteln können, sind gewisse, wenn auch vielleidht verhältnismäßig geringe Lichtstärken erforderlich, und sicherlich werden die Tiere bei etwas größeren Lichtstärken innerhalb gewisser Grenzen besser unterscheiden und sehen können als bei minimalen. Die Motte, die an den finstersten Stellen von Wald und Gebüsch nichts oder nicht genügend sieht, wird, wenn sie einer von Mond-oder Sternenlicht wenn auch nur schwach erhellten Stelle zufliegt, mit Hilfe ihrer Augen leichter die Artgenossen finden, den Feind fliehen oder ihrer Nahrung nachgehen können, und es ist wohl verständlich, wenn ein Drang, der den Tieren in der Mehrzahl der Fälle von größtem Vorteile ist, so lebhaft werden kann, daß sie ihm auch in jenem für sie ungewohnten, ja fremden 
Falle folgen, wo er ausnahmsweise nicht von Nutzen ist oder gar den Tod bringt.

Eine solche Betrachtungsweise führt, wie ich meine, auch zur Beantwortung jener anderen, oft erörterten Frage, warum die Insekten, die so lebhaft zum Lichte gehen, nicht auch in die Sonne oder in den Mond fliegen. Wenn wir im halbdunklen Zimmer einen Gegenstand deutlicher unterscheiden wollen, so treten wir mit ihm ans Fenster oder an die Lampe heran. Niemand aber wird, wenn er den Gegenstand im Freien, etwa bei schon tief stehender Sonne deutlicher sehen will, nach Westen gehen, um der Sonne näher zu sein. Um nicht in den Verdacht unzuläßigen „Anthropomorphisierens" zu kommen, drücke ich das Gesagte mehr physikalisch aus: Wenn die Motte des Nachts aus dem Schatten eines Baumstammes auf eine vom Mondlichte getroffene Stelle oder nach einer wenige Meter entfernten Flamme fliegt, gelangt sie beidemale in Gebiete größerer Lichtstärke und dementsprechend nehmen die von ihr wahrgenommenen Helligkeiten merklich zu. Für das Insekt aber, das eine Strecke weit auf Sonne oder Mond zufliegen wollte, würde sein relativer Abstand von dieser Lichtquelle sich viel $z u$ wenig ändern, als daß Lichtstärke und Helligkeit dabei merklich zunehmen könnten.

Zeigen uns diese Erwägungen den Weg, auf dem jener scheinbare Widerspruch im wesentlichen seine Lösung findet, so darf auch nicht vergessen werden, daß die Bewegungen der fraglichen Insekten nicht ausschließlich von dem zu ihnen gelangenden Lichte bestimmt werden, letzteres vielmehr im allgemeinen nur einen von mehreren gleichzeitig auf die Tiere wirkenden Faktoren bildet. Und wie die so lebhaft zum Hellen schwimmenden Fische bei ihren Bewegungen zum Lidhte in der Regel nicht aus ihrem gewohnten Medium, ja vielfach nicht einmal bis dicht zur Wasseroberfläche geführt werden, so dürften auch. z. B. bei den meist in der Nähe von Erde, Bäumen und Sträuchern fliegenden Motten neben den vorhin angeführten Umständen auch die Beziehungen zu dieser ihnen gewohnten Umgebung ihre Flugrichtung mehr oder weniger mit beeinflussen.

Unter den fliegenden Insekten, die ich untersuchte, und die vielfach ein ähnliches Verhalten zeigen, wie die bisher be- 
sprochenen Wirbellosen, seien die Bienen etwas eingehender besprochen, weil man ihnen heute noch allgemein einen vorzüglichen Farbensinn zuschreibt. Auch sie eilen, wenn ich sie in einem geeigneten Behälter ins Spektrum bringe, aus dem rot und aus dem blau und violett durchstrahlten Teile zum Gelbgrün bis Grün, hier laufen sie senkrecht nach oben. Wird die eine Hälfte ihres Behälters mit rotem, die andere mit blauem Glaslichte bestrahlt, so laufen die Tiere lebhaft nach dem Blau, auch wenn dieses unserem Auge weniger hell erscheint, als das Rot. Erst wenn die rote Hälfte so lichtstark gemacht wird, daß sie für uns bedeutend heller erscheint als die blaue, machen die Bienen Kehrt und eilen aus dem Blau ins Rot; dazwischen kann leicht ein solches Lichtstärkenverhältnis beider Lichter ermittelt werden, wo die Bienen keines von beiden bevorzugen, sondern sich annähernd gleichmäßig in der roten und in der blauen Hälfte verteilen.

Frühere Untersucher haben mehrfach angegeben, Blau sei die "Lieblingsfarbe" der Bienen; Versuche, wie die eben geschilderten, genügen schon, um eine solche Annahme zu widerlegen; aber die Meinung, daß die Bienen einen vorzüglichen, dem unseren vergleichbaren Farbensinn hätten, ist noch weit verbreitet.

Diese Meinung geht im wesentlichen auf $C h$. D. Sprengel zurück, der vor 120 Jahren lehrte, die Insekten würden aus der Ferne von der Farbe der ganzen Blume angezogen, in der Nähe zeigten farbige Streifen und Flecken ihnen den Weg zu den Honigbehältern. Sprengels Auffassung wird heute noch von den meisten Zoologen und Botanikern geteilt; sie schien eine befriedigende Erklärung für das Auftreten farbiger Blüten zu geben, und so vergaß man, daß jener ohne alle Kenntnis vom Lichtsinne der Bienen aufgestellten Hypothese jede wissenschaftliche Begründung fehlt.

Sprengels Lehre bildet auch heute noch die wesentliche Grundlage für die Annahme eines Farbensinnes bei Bienen; wiederholt wurde versucht, diese durch das Experiment zu stützen, insbesondere auch durch die oft wiederholte Angabe, es gelinge, Bienen auf bestimmte Farben $z u$ "dressieren“. Aber die Un- 
haltbarkeit der herrschenden Lehre von der Bedeutung der Blumenfarben für den Bienenbesuch ist schon genügend dargetan durch den von mir erbrachten Nachweis, daß das Vorhandensein eines dem unsrigen auch nur entfernt ähnlichen Farbensinnes bei Bienen ausgeschlossen ist. Die Annahme einer anziehenden Wirkung der Blumenfarben setzt voraus, daß letztere den Bienen wenigstens bis zu einem gewissen Grade ähnlich erscheinen müßten, wie uns; denn wenn man ihnen farbige Sehqualitäten zuschreibt, von denen wir uns keine Vorstellung machen können, so entfällt die Berechtigung zu der Annahme, die für uns schön farbigen Blüten müßten auch den Bienen so erscheinen.

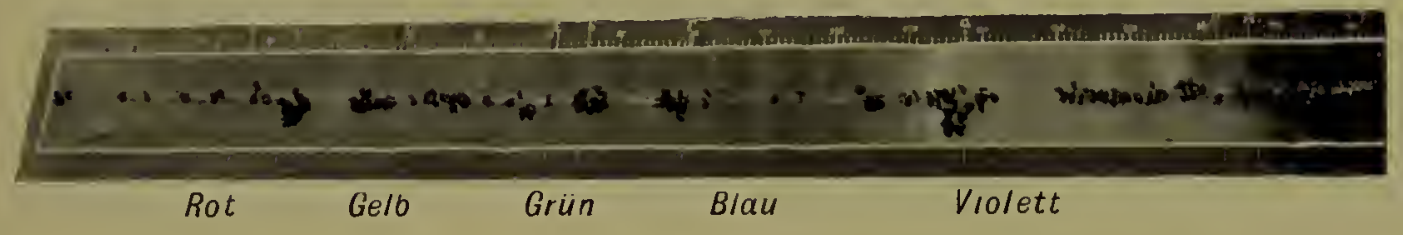

Fig. 11.

Bienen, die durch mehrere Tage auf Gelb „dressiert" waren, suchen Honig auf dem mit einem Honigstreifen versehenen Spektrum. Die Tiere haben sich angenähert gleichmäßig im ganzen Spektrum verteilt, das Gelb, auf das sie dressiert waren, wird $\mathrm{nicht}$ stärker besucht als die übrigen Farben.

Meine Befunde über den geringen Helligkeitswert roter Lichter für die Bienen werden heute schon von solchen Autoren anerkannt, die sonst noch ganz auf dem alten Standpunkte der S p r en g e lschen Hypothese verharren zu können glauben: Dressurversuche führten diese Autoren zu dem nach meinen früheren Befunden selbstverständlichen Ergebnisse, daß die Bienen Rot mit Schwarz, Purpurrot mit Blau und Violett verwechseln. Daraus läßt sich doch nur der eine Schluß ziehen, daß bei allen mehr oder weniger roten, also den gelbroten, roten und blauroten Blumen die Farben nicht um der lnsekten willen da sind; könnten doch alle roten Blumen ebensogut schwarz, alle bläulichroten und violetten ebensogut blau sein, ohne darum anders auf die Bienen zu wirken. Muß man nun schon zugeben, daß bei dieser ganzen großen Gruppe der mehr oder weniger rot blühenden Pflanzen eine Beziehung ihrer Blütenfarbe zum Bienenbesuche ausgeschlossen ist, so scheint es nicht unbedenklich, für die gelben und blauen 
Blüten anzunehmen, sie allein seien um der Insekten willen gefärbt, nicht aber die roten. Diese heute noch nachdrücklich vertretene Annahme stützt sich auf die Angabe, es gelinge, Bienen zwar nicht auf rote, wohl aber auf gelbe und blaue Papiere zu "dressieren". Stellt man aber solche Versuche unter genügender Berüdksichtigung aller in Betracht kommenden Fehlerquellen an, wie ich dies vielfach getan habe, und trägt man insbesondere Sorge, daß die Ansammlungen der Bienen an bestimmten Stellen nicht durch andere Umstände, wie z. B. den Geruchssinn, mit beeinflußt werden, so kann man mit den von mir entwickelten Methoden leicht eindringlich zeigen, daß es ganz unmöglich ist, Bienen auf irgendeine Farbe zu dres. sieren. Damit fällt die einzige Stütze, die die Anhänger der Sprengelschen Lehre zu deren Gunsten ins Feld führen konnten. -

Aus einer großen $Z$ ahl von mir angestellter Versuche sei nur einer zur Erläuterung des Gesagten angeführt: Eine ca. $2 \mathrm{~m}$ breite, aus 185 verschiedenen frei farbigen Papieren passend zusammengestellte Fläche (Fig. 11) zeigt die verschiedenen Farben des Spektrums in allen Übergängen; sie wird, mit einem schmalen Honigstreifen versehen, in der Nähe eines Stodkes aufgestellt, dessen Bienen durch mehrere Tage auf Gelb „dressiert" worden waren und die daher nach der herrschenden Lehre sich selbst dann im Gelb sammeln sollten, wenn ihnen hier kein Honig geboten wird. Die photographische Aufnahme zeigt, daß davon keine Rede ist; die auf Gelb dressierten Bienen verteilen sich angenähert gleichmäßig in allen Farben des Spektrums und bevorzugen selbst dann nicht das Gelb, wenn ihnen auch hier Honig geboten wird.

Von botanischer Seite wurde mir wiederholt gesagt, man könne meine Anschauungen nur um deswillen nicht anerkennen, weil dann die Erklärung für das Auftreten bunter Blütenfarben fehle. Man darf aber nicht übersehen, wie vielfach im Pflanzenreiche bunte Farben sich auch da finden, wo von einer Beziehung zum Insektenbesuche keine Rede sein kann. Man denke an das bekannte Auftreten schön gelber und roter Farben bei windbestäubten Pflanzen, an die roten Blätter versdhiedener Bäume, an die mannigfaltigen, zum Teile wundervollen Färbungen zahlreicher Flechten, die nach Angabe der maßgebenden Fachmänner nichts mit Insektenbesuch zu tun haben; man erinnere sich auch der lebhaften Farben mancher dauernd unterirdischer Pflanzen- 
teile, wie sie unter anderen bei verschiedenen Rüben- und Retticharten vorkommen. Wenn bunte Farben im Pflanzenreiche so häufig vom Insektenbesuche unabhängig auftreten, erscheint es nicht logisch, für die bunten Farben der Blüten ohne weiteres anzunehmen, sie könnten nur um der Insekten willen zur Entwicklung gekommen sein.

Im Hinblicke auf die hier kurz angedeuteten, heute viel erörterten Fragen war ich bemüht, weitere neue Methoden zum Studium des Lichtsinnes der Bienen zu entwickeln, mit deren Hilfe auch der Laie sich leicht von der Unhaltbarkeit der herrschenden Anschauungen über Farbensinn bei Bienen überzeugen kann. Auch die so erhaltenen, unschwer nachzuprüfenden Ergebnisse sind mit der Annahme eines wie immer gearteten Farbensinnes der Bienen unvereinbar, dagegen ohne weiteres verständlich, ja zu erwarten, wenn diese total farbenblind sind. Der pflanzenbiologischen Forschung erwachsen aus den neuen Befunden neue interessante Aufgaben. -

Nur kurz sei jener Tiere gedacht, die, ohne nachweisliche Sehorgane zu besitzen, deutliche Lichtreaktionen zeigen. Als Beispiel diene die $\mathrm{S}$ and mus chel (Psammobia vespertina). Das Tier streckt, wenn es ungestört im Sande liegt, zwischen den Schalen eine weißliche Röhre, den Sipho, hervor, der bei plötzlicher Belichtung zurückgezogen wird, um so stärker, je größer ceteris paribus die Lichtstärke des Reizlichtes ist. Bringe ich die Tiere ins Spektrum, so verkürzt sich bei Belichtung mit Rot der Sipho nur wenig, bei Belichtung mit Gelbgrün bis Grün weitaus am stärksten, bei Belichtung mit Blau weniger stark als im Grün, aber wesentlich stärker als im Rot. Also auch für diese Gebilde, an welchen anatomisch bisher keinerlei Sehorgane, ja nicht einmal besondere lichtempfindliche Zellen nachgewiesen werden konnten, haben die verschiedenen homogenen Lichter ähnliche oder die gleichen relativen Reizwerte, wie für das total farbenblinde Menschenauge. Ein gleiches konnte ich für verschiedene Arten von Würmern dartun. -

Man hat bisher angenommen, die Reaktionsfähigkeit der lichtempfindlichen Substanz der Sehorgane gegenüber Strahlen verschiedener Wellenlänge sei bei verschiedenen Wirbellosen 
wesentlich verschieden, so, daß auch die Grenzen des für sie in Betracht kommenden Spektrums bei verschiedenen Arten beträchtliche Verschiedenheiten zeigen könnten. Meine Untersuchungen ergeben demgegenüber, daß bei allen bisher darauf untersuchten Wirbellosen die Grenzen des Spektrums jedenfalls annähernd dieselben und keine wesentlich anderen sind, als beim total farbenblinden Menschen. Dieser Befund scheint mir von besonderem Interesse; lehrt er doch, daß trotz so großer Verschiedenheiten der Lebensbedingungen, wie sie z. B. die in Luft und die in Wasser lebenden Wirbellosen zeigen, hier das nervöse Sehorgan bei Übergang vom Wasser- zum Luftleben keine Änderung seines Verhaltens gegenüber Strahlen verschiedener Wellenlänge im Sinne einer Anpassung bzw. andersartigen Verwertung der verschiedenen Strahlen erfahren hat.

Und doch ist ein gewisses "Hinausrücken" der Sichtbarkeitsgrenzen des Spektrums bei manchen Wirbellosen erfolgt, freilich auf ganz anderem, als dem bisher angenommenen Wege, nämlich vermittels der Fluoreszenz.

Fluoreszierende Körper besitzen bekanntlich die Eigentümlichkeit, Strahlen von verhältnismäßig kleiner Wellenlänge in solche von größerer Wellenlänge umzuwandeln. Insbesondere zeigt eine Reihe organischer Gebilde das Vermögen, kurzwellige (violette und ultraviolette) Strahlen in solche von mittleren Wellenlängen umzuwandeln, sie fluoreszieren mit grünlichem (gelblichgrünem bis bläulichgrünem) Lichte. Ich fand nun die interessante Tatsache, daß eine derartige Fluoreszenz in überraschend hohem Maße unter anderem den Kristallkegeln des Arthropodenauges zukommt. Eine Folge davon ist, daß Strahlen, die an sich dem betreffenden Auge nur sehr wenig hell erscheinen, eine viel beträchtlichere Helligkeitsempfindung auszulösen vermögen, indem sie mittels Fluoreszenz in Strahlen von mittlerer Wellenlänge verwandelt werden, wodurch also ein vorwiegend grünes Licht entsteht, das, wie ich früher zeigte, für die fraglichen Augen von allen homogenen Strahlungen den größten Helligkeitswert besitzt.

Ich fand weiter, daß bei Krustern nicht nur die Augen, sondern auch der ganze Panzer im violetten und ultravioletten Lichte fluoresziert und konnte ein gleiches auch für die Körper- 
oberfläche junger Aale nachweisen. Unter den Coelenteraten fand ich die inneren, der Mundhöhle näher gelegenen Tentakel mit einer Reihe kleiner Fledke besetzt, die eine ungemein lebhafte Fluoreszenz zeigen, so daß sie z. B. in tief dunkel blauem Lichte als leuchtend grüne Punkte hervortreten. Noch kennen wir die Lebensgewohnheiten der genannten Tierarten zu wenig, um bereits angeben zu können, welche biologische Bedeutung diesen Fluoreszenzerscheinungen zukommen mag; daß es sich insbesondere in den zuletzt genannten Fällen um rein zufällige Erscheinungen handle, ist wohl wenig wahrscheinlich angesichts der Tatsache, daß in jenen Meerestiefen, wo infolge der relativ starken Absorption insbesondere der langwelligen Strahlen die herrschende Gesamthelligkeit schon ziemlich gering ist, eben jene kurzwelligen Strahlen vorwiegen, die für die Fluoreszenzerregung hauptsächlich in Betracht kommen.

Wenn immer Physiologen und Zoologen ihr Interesse der vergleichenden Erforschung der Lebensvorgänge bei niederen Tieren zuwandten, zeigte sich ein Bestreben, „die unendlich kunstreiche, aber doch genau beschränkte Technik jener Geschöpfe für ganz maschinenmäßig" zu erklären; und auch heute sehen wir verdiente Forscher nachdrüdklich die Meinung vertreten, es seien die lebenden Organismen nichts anderes als chemische Maschinen. Prüfen wir, ob aus den neuen Tatsachen, die wir über das Verhalten der Tiere zum Lichte kennen lernten, sich uns neue Gesichtspunkte ergeben, die eine Stellungnahme im Streite der Meinungen gestatten.

Nehmen wir an, es gelänge, z. B. mit Hilfe von Selenzellen, Maschinen herzustellen, die bei Belichtung sich in Bewegung setzten und bei Einwirkung zweier Lichtquellen sich in der Richtung nach der lichtstärkeren von beiden bewegten. Nehmen wir weiter an, es ließe sich, etwa durch passende lichtabsorbierende Farbstoffe, eine besonders starke Wirkung der gelbgrünen bis grünen Strahlen des Spektrums auf diese Maschinen erreichen und man lernte auch Stoffe herzustellen, die bei Belichtung allmählich verbraucht würden und im Dunkel sich erneuten, so daß 
sie wenigstens innerhalb gewisser Grenzen adaptative Änderungen nachzuahmen gestatteten: Wir hätten dann auf Licht reagierende Maschinen, die im Spektrum nach der Gegend des Gelbgrün bis Grün gingen, ganz so wie jene sogenannten positiv phototropischen Tiere. Wären wir aber damit im Verständnisse der Lebensvorgänge und des Sehens bei den zum Lichte gehenden Organismen im geringsten gefördert? Ich glaube nicht. Bliebe doch gerade das unerklärt, was an unseren neuen Befunden das Wesentliche, zugleich die merkwürdigste von allen jenen Erscheinungen ist, die Übereinstimmung der relativen Reizwerte verschiedenfarbiger Lichter für die Sehorgane jener niederen Lebewesen mit den Helligkeitswerten, die diese Lichter für das total farbenblinde und für das dunkeladaptierte normale Menschenauge haben.

Solange wir in jenen Lebewesen nur empfindungslose chemische Maschinen sehen, stehen wir vor einem Wirrwarr zusammenhangloser Tatsachen, die durch die Übereinstimmung mit den Eigentümlichkeiten der Helligkeitsempfindung im Menschenauge nur wunderbarer, nicht verständlicher werden.

Nehmen wir aber an, daß dem im wesentlichen ähnlichen Organe bei den Tieren auch eine im wesentlichen ähnliche Funktion entspricht, daß also den vom Lichte im Sehorgane ausgelösten physischen Regungen nicht nur beim Menschen, sondern auch bei jenen niederen Tieren psychische Korrelate zugehören, so kommen wir in die Lage, die aus den Bewegungen erschlossenen psychischen Vorgänge oder Sehqualitäten bei den Tieren mit den an unserem eigenen Sehorgan durch die nämlichen Lichtreize ausgelösten zu vergleichen. Der von mir erbrachte Nachweis der zwischen beiden bestehenden Beziehungen, Ähnlichkeiten und Verschiedenheiten, gestattet jetzt, das Studium der Sehqualitäten bei niederen Tieren in ganz ähnlicher Weise und zum Teile mit fast der gleichen Genauigkeit experimentell und rechnerisch in Angriff zu nehmen, wie bei unserem eigenen Sehorgan, und es ist so zum ersten Male möglich geworden, nicht nur die beobachteten Bewegungen der Tiere befriedigend zu erklären, sondern auch die unter beliebig gewählten Versuchsbedingungen zu erwartenden mit Bestimmtheit vorauszusagen. 
$\mathrm{Daß}$ so mächtigen und so verbreiteten Lebensäußerungen, wie es die durch das Licht in der Tierreihe hervorgerufenen Bewegungen sind, auch eine große biologische Bedeutung zukommen muß, werden Viele als selbstverständlich einer Erwähnung kaum für bedürftig halten. Aber die Anhänger der Lehre, die in den Organismen nur empfindungslose chemische Maschinen sieht, vertreten auch die Meinung, jene Bewegungen zum Lichte seien für das Tier im allgemeinen weder nützlich noch schädlich; wenn sie auch gelegentlich den Tieren von Nutzen sein könnten, seien sie doch nicht durch die biologischen Interessen der Tiere diktiert. Man begründet eine solche Stellungnahme unter anderem durch den Hinweis auf die vielen Insekten, die diesem Triebe zum Opfer fallen, indem sie des Nachts in die Flamme fliegen. Dem ist aber entgegenzuhalten, daß, bei niederen wie bei höheren Tieren, wohl überhaupt keine Lebensäußerung sich zeigt, die ausnahmslos und unter allen Bedingungen der Art nur zum Vorteile gereichte: Die durch Hunger und durch Liebe bestimmten Lebensäußerungen in der Tierreihe sind für die Erhaltung der Art von der höchsten Bedeutung, und doch, wie groß ist die Zahl der Individuen, die eben durch sie unter besonderen Verhältnissen zur Vernichtung geführt werden!

Wenn die Pflanze sich zur Sonne wendet, sehen wir darin wohl alle nicht sowohl eine gewissermaßen zufällige Reaktion, als vielmehr den Ausdruck der Bedeutung des Lichtes für ihr Wachstum und ihre Entwicklung. Soll eine derartige Betrachtungsweise nur für die eine Gruppe der Lebewesen Berechtigung haben, die unter dem Einflusse des Lichtes heranwachsen, nicht aber für die Tiere? Und wie ließe sich Entwidklung und Verbreitung eines so elementaren Dranges verstehen, wenn er ohne Bedeutung für die Erhaltung der Arten wäre, die ihn zeigen? -

Die Auffassung, die auch den niederen Tieren eine Helligkeitsempfindung zuschreibt, erscheint wohl begründet durch unsere Messungen, welche die weitgehende Übereinstimmung zwischen den Lichtreaktionen der Tiere und den Helligkeitswahrnehmungen des total farbenblinden Menschenauges in ihrer Abhängigkeit von der Wellenlänge des Lichtes erkennen lassen. 
Diese Erkenntnis führt uns auch zu klareren Vorstellungen über Art und Entwidklung des Lidhtsinnes in der Tierreihe.

Unter der spezifis chen Energie einer Sinnesnervensubstanz verstehen wir die besondere Eignung dieser Substanz zu ganz bestimmten physischen Vorgängen, denen ebenso bestimmte psychische Phänomene entsprechen. Das physiologische Experiment hat uns nun dazu geführt, schon jener einzelnen Lichtsinnzelle, der wir beim Lanzettfische begegneten, die spezifische Energie zuzuerkennen, auf bestimmte Lichtreize mit der Vermittlung bestimmter Helligkeitsempfindungen zu antworten. Von der Art dieser letzteren haben wir jetzt durch Experiment und Messung für eine Reihe von Tierarten ziemlich genaue Kunde.

Lange Zeit hindurch, so müssen wir wohl annehmen, war das Leben der Tierwelt auf das Wasser beschränkt; und so mannigfach verschieden Bau und Entwidklung des äüßeren Auges der Wassertiere ist, bei allen zeigen die vom nervösen Sehorgane vermittelten Lichtreaktionen im wesentlichen die gleiche Abhängigkeit von der Wellenlänge, in der wir eine so wundervolle Anpassung an die besonderen Bedingungen des Wasserlebens kennen lernten.

Erst mit dem Übergange zum Luftleben konnte die Entwidklung eines Farbensinnes für die tierischen Lebewesen von wesentlicher Bedeutung werden. Indessen zeigen, wie wir sahen, die bisher von mir untersuchten in Luft lebenden Wirbellosen in den hier in Betracht kommenden Beziehungen ähnliches oder das gleiche Verhalten ihres Lichtsinnes, wie die Wassertiere und wie der total farbenblinde Mensch; hier, bei den Wirbellosen, hat also der Übergang zum Luftleben nicht auch zur Entwidklung der Fähigkeit der Farbenempfindung geführt. Während man bisher einen Farbensinn in der Tierreihe weit verbreitet glaubte, zeigen meine Untersuchungen, daß ein solcher allein bei den in Luft lebenden Wirbeltieren zur Entwidklung gekommen ist.

Man hat geglaubt, gegen die Lehre von den spezifischen Sinnesenergieen den Einwand erheben zu können, sie setze die Unveränderlichkeit der Funktionen eines Sinnesorganes voraus und widerspreche damit dem Entwidklungsgedanken. Daß Johannes Muellers Lehre nicht in diesem Sinne auszu- 
legen, daß sie vielmehr einer weiteren, umfassenderen Deutung und Anwendung zugängig ist, hat $\mathrm{Ewald} \mathrm{Hering}$ in klassischen Ausführungen gezeigt; ein besonders schönes und eindringliches Beispiel für deren Richtigkeit scheint mir eben das zu sein, was uns die vergleichende Physiologie über die stammesgeschichtliche Entwicklung von Licht- und Farbensinn gelehrt hat: Die spezifischen Energieen der nervösen Substanz des Sehorgans, die bei den wasserlebenden tierischen Organismen in überall gleicher Weise nur die Empfindung farbloser Helligkeiten vermittelten, haben bei den Wirbeltieren mit dem Übergange zum Luftleben unter dem Einflusse der so viel größeren Mannigfaltigkeit der nunmehr zum Sehorgan gelangenden Strahlungen eine Umbildung erfahren, vermöge deren sie jetzt neben den farblosen Helligkeiten auch die bunten Farben zum Bewußtsein bringen; nicht so, da $ß$ an Stelle der früheren nunmehr gan $z$ andere Energieen getreten wären, die jene ersten gewissermaßen abgelöst und verdrängt hätten: Kehren doch die letzteren selbst beim Menschen nicht nur bei jener merkwürdigen Störung wieder, die wir vorhin als totale Farbenblindheit kennen lernten; auch in jedem normalen Auge treten, sobald wir, nach genügender Herabsetzung der Lichtstärke, uns an das umgebende Dunkel anpassen, die ursprünglichen, uralten spezifischen Energieen wieder hervor, welche wir weit herab in der Tierreihe, ja selbst da noch nachweisen konnten, wo die Wahrnehmung von Licht noch nicht durch ein eigentliches Auge vermittelt wird.

So treu bewahrt unser Sehorgan die Erinnerung an das, was es einst, vor unermeßlich langen Zeiten, gewesen, als ihm die bunte Welt der Farben noch verhüllt war. 
Drudk der Königl. Universitätsdruckerei H. Stürtz A. G., Würzburg 